\title{
Intraspinal intradural nodular fasciitis mimicking glioblastoma metastasis: a case report
}

\author{
Carl Moritz Zipser ${ }^{1,2}$, Lars Füllbier ${ }^{3,4}$, Rudi Beschorner ${ }^{4,5}$, Hans Bösmüller ${ }^{6}$, Ulf Ziemann ${ }^{1,2}$, \\ Marcos Soares Tatagiba ${ }^{3,4}$, Ghazaleh Tabatabai ${ }^{1,2,4,5,7,8}$, Benjamin Bender ${ }^{4,9}$, Felix Behling B $^{3,4}$ \\ ${ }^{1}$ Department of Neurology and Stroke, University of Tuebingen, Tuebingen, ${ }^{2}$ Hertie Institute for Clinical Brain Research, \\ University of Tuebingen, Tuebingen, ${ }^{3}$ Department of Neurosurgery, Eberhard-Karls University, Tuebingen, ${ }^{4}$ Comprehensive Cancer \\ Center Tuebingen, Eberhard-Karls University, Tuebingen, ${ }^{5}$ Department of Neuropathology, Eberhard-Karls University, Tuebingen, \\ ${ }^{6}$ Department of Pathology, Eberhard-Karls University, Tuebingen, ${ }^{7}$ Interdisciplinary Division of Neuro-Oncology, Departments \\ of Vascular Neurology and Neurosurgery, Eberhard-Karls University, Tuebingen, ${ }^{8}$ Center for Personalized Medicine, Eberhard-Karls \\ University, Tuebingen, ${ }^{9}$ Department of Diagnostic and Interventional Neuroradiology, Eberhard-Karls University, Tuebingen, Germany
}

\begin{abstract}
We report the case of a 78-year-old male patient suffering from right temporal glioblastoma with radiographic meningeal tumor spread. During the further course of the disease he developed a rapidly progressive paraplegia. An magnetic resonance imaging scan showed a contrast enhancing an intraspinal intradural lesion with compression of the myelon on segment Th 8/9. With a high suspicion of a spinal metastasis of the known glioblastoma, emergency spinal decompression and resection of the intradural mass was performed. However, histopathological evaluation revealed nodular fasciitis without any signs of glial origin.
\end{abstract}

Key words: glioblastoma, spinal metastasis, nodular fasciitis, acute paraplegia, myelon compression.

\section{Introduction}

Nodular fasciitis (NF) is a rapidly growing but non-malignant lesion. It is self-limiting and usually regresses over a few months but due to its rapid growth and unspecific imaging characteristics it is often misinterpreted as a malignant process, mostly as a sarcomatous lesion. The cause of this fibroblastic/myofibroblastic proliferation is unclear while a possible correlation with local trauma has been suggested. NF preferably develops in the upper extremities, trunk, head and neck, but it can also occur in many other locations $[2,4,7]$. Only two cases with a intraspinal extradural location have been reported in the literature $[5,6]$ and one case with intracranial intradural manifestation [1]. This case report is the first description of an intraspinal intradural nodular fasciitis. It occurred in a patient with right temporal glioblastoma and radiographic meningeal tumor spread.

\section{Case description}

A 77-year-old male patient presented to the Department of Neurology with a two-month histo- 
ry of progressive headache and nausea. The neurologic examination revealed neck stiffness, afferent gait disturbance and neuropsychological deficits (i.e. disorientation in time, psychomotor retardation), but no sensorimotor deficits. Extensive radiologic and cerebrospinal fluid workup was done. Magnetic resonance imaging (MRI) scans showed a rightsided multicentric temporomesial contrast-enhancing lesion (Fig. 1A-B) as well as marked leptomeningeal tumor spread in cranial and spinal images (Fig. 1C-D). A navigated right temporal biopsy was done which led to the diagnosis of glioblastoma, isocitrate dehydrogenase (IDH)-wildtype, WHO grade IV (Fig. 2A-C). The $\mathrm{O}^{6}$-methylguanin-methyltransferase (MGMT) promotor was unmethylated. Treatment was composed of computed tomography (CT)-based whole-brain photon-radiotherapy including cervical vertebra II and concomitant temozolomide chemotherapy $\left(75 \mathrm{mg} / \mathrm{m}^{2}\right.$ body surface area). One month after completion of radiotherapy, the patient presented to the department of neurosurgery with acute painless paraplegia and complete loss of sensory sensations of the lower extremities. Spinal MRI indicated a novel intradural lesion at the level of thoracic vertebrae $8 / 9$ with significant compression of the spinal cord (Fig. 1E-F) when compared with previous spinal images 3 months earlier (Fig. 1D). Emergency laminectomy of thoracic vertebrae 7-9 and partial tumor resection was performed (Fig. 1G-H). The detailed histology of this lesion is discussed in the section pathology report (Fig. 2D-F). In the medium term unfortunately no patient recovery was achieved, related to the severe affection of the spinal cord and additional diagnosed critical illness polyneuropathy. Due to the poor condition, further causal therapy was not initiated in a process of shared decision-making.

\section{Pathological findings}

The resected tissue of the spinal lesion consisted of several pieces of tissue with a grayish color and a moderate to firm consistency, which in total had a diameter of $1.0 \mathrm{~cm}$.

Histology showed a spindle-shaped lesion with moderate cell density and low to moderate nuclear pleomorphism. Focally microvascular proliferations and an epithelioid-like cell morphology were noted (Fig. 2D). There were only very few mitoses restricted to microvascular proliferations. Numerous thin collagenous fibers and in some areas also numerous reticulin fibers were intermingled. The MIB-1 proliferation index was approximately $10 \%$ and MIB1-positive cells were often seen along microvascular proliferations (Fig. 2E). In some areas interspersed CD163-positive macrophages were seen in moderate density and an expression of smooth muscle actin was notable. There was no specific expression of actin (clone HHF35), calponin, glial fibrillary acidic protein (GFAP) (Fig. 2E), STAT6, melan A, cytokeratin pan antibody (pan-CK), epithelial membrane antigen (EMA), progesterone-receptor and neurofilament. S100 was only focally expressed and CD34 staining was restricted to blood vessels. Thus, a metastasis of the known glioblastoma could be excluded. Additionally, a FISH analysis was performed but failed to show a clear USP6 rearrangement. The lesion was considered to be a reactive proliferation, consistent with a nodular fasciitis.

\section{Discussion}

This patient with a history of a right temporal glioblastoma developed a reactive intraspinal lesion. Although we could not demonstrate an USP6 rearrangement, a characteristic that is often but not always found in nodular fasciitis [3], we assume nodular fasciitis to be the most likely diagnosis. Another marker that is typically positive in nodular fasciitis is calponin. However, its negativity could not exclude the diagnosis. Importantly, a solitary fibrous tumor was unlikely, given STAT6 negativity. To the best of our knowledge, this is the first report of an intraspinal and intradural nodular fasciitis. The fact that it occurred in a patient who is suffering from a right temporal glioblastoma with radiographic meningeal tumor spread suggests a histopathological connection. However, reviewing the histopathological characteristics of the temporal glioblastoma, no mesenchymal aspects, as found in gliosarcomas, were appreciated. Additionally, the intraspinal intradural lesion showed no signs of glial origin, thus ruling out spinal glioblastoma metastasis. One can only speculate if the formation of an intradural nodular fasciitis was triggered by tumor spread which was radiologically suspected. It is known that NF can arise from many different tissue types that contain fibroblasts, ranging from skeletal muscle and fascia to dermis, subcutaneous tissue and blood vessels. Recent localized trauma has been the only suggested 

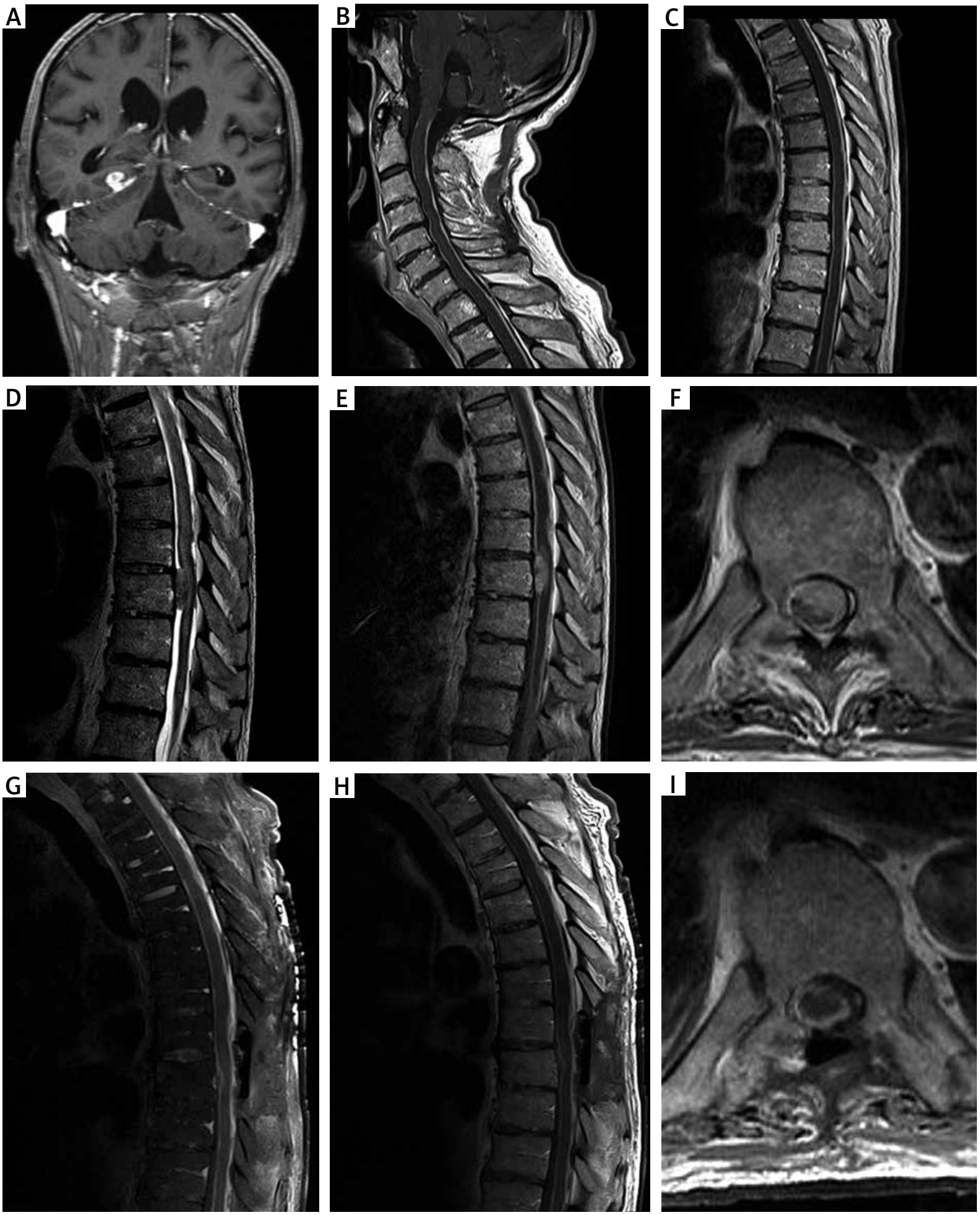

Fig. 1. Right-sided multicentric temporomesial contrast-enhancing lesion on post-contrast T1 sequences (A, B) with spinal leptomeningeal tumor spread (C, D). Three months later, while the patient presented with paraplegia, a novel intradural lesion at the level Th8/9 with myelon compression was seen on spinal MRI post-contrast T1 and T2 sequences (E, F). The postoperative images showed a good decompression of the myelon and partial resection in post-contrast T1 and T2 sequences $(G, H)$. 

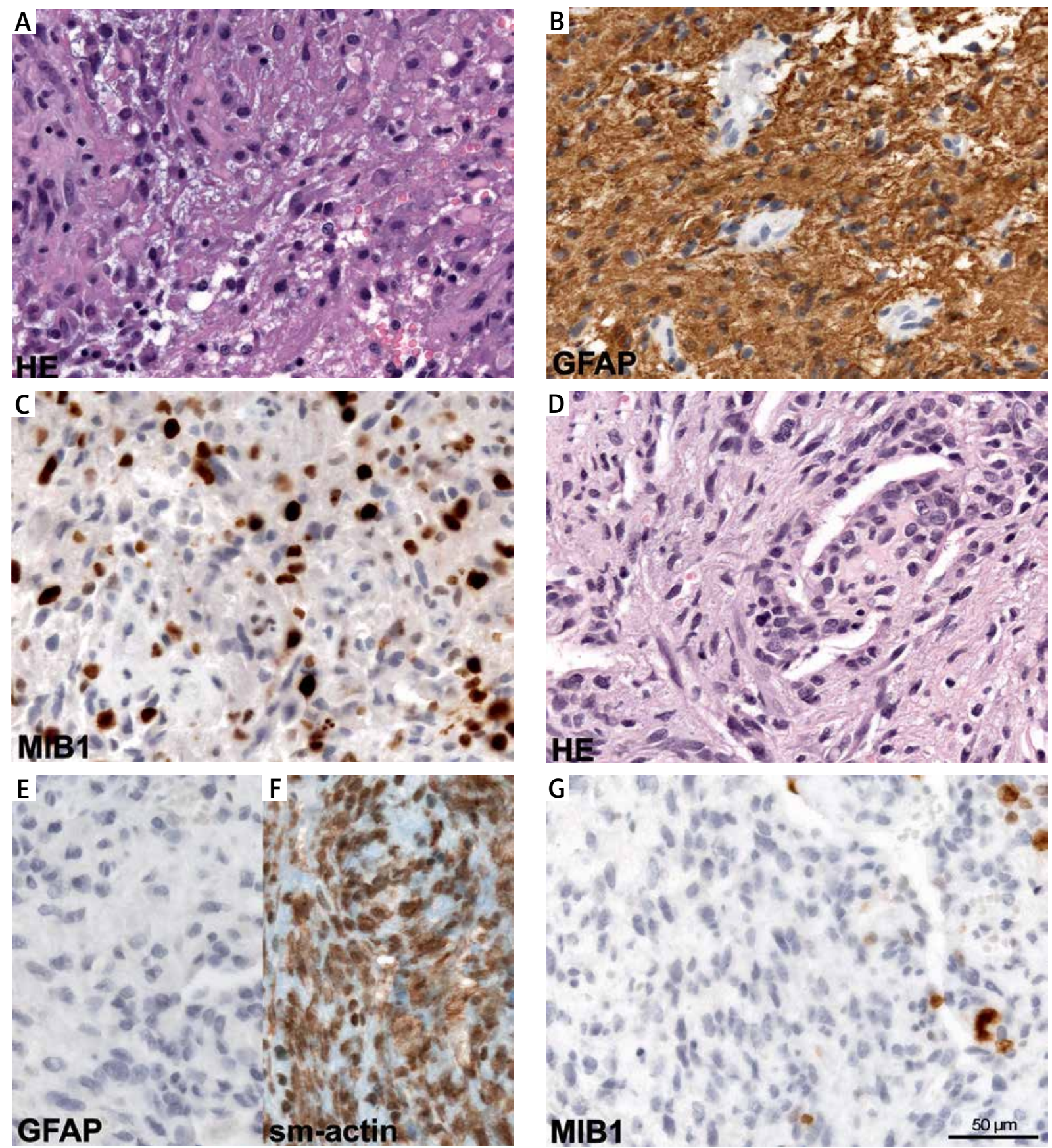

Fig. 2. The temporal tumor (A-C) showed tumor cells on a fibrillary background with an astrocytic morphology, distinct expression of the glial fibrillary acid protein (GFAP) and numerous MIB1-positive cells. Additionally, necrosis and few endothelial proliferations were found (not shown). In contrast, the spinal lesion consisted of spindle shaped cells. Epithelioid cells were noted in the vicinity of microvascular proliferations (D). This lesion lacked expression of GFAP (E), was immunoreactive for smooth muscle actin (F) and showed moderate numbers of MIB1-positive cells, preferentially along microvascular proliferations (G). 
possible factor correlated with NF in 10 to $15 \%$ [4]. Even though mechanical or inflammatory irritation of the meninges by meningeal glioblastoma spread seems conceivable, the true pathogenesis in this special case remains unclear.

\section{Conclusions}

This is the first reported case of an intraspinal intradural nodular fasciitis. The case illustration emphasizes the difficulties and misinterpretation that can occur with the clinical and radiographic presentation and the importance of a comprehensive and accurate histopathological workup. Regardless of the benign nature of the disease, timely surgical excision of an intraaxial nodular fasciitis is recommended to prevent or alleviate neurological deficits.

\section{Acknowledgments}

The authors would like to thank Ulrich Vogel (Department of Pathology, Eberhard-Karls University, Tuebingen, Germany) for the FISH analysis on a USP6 rearrangement.

\section{Disclosure}

The authors report no conflict of interest.

\section{References}

1. Agozzino M, Cavallero A, Inzani F, Acchiardi I, Locatelli D, Scagnelli P, Malattia C, Arbustini E. Cranial fasciitis with exclusive intracranial extension in an 8-year-old girl. Acta Neuropathol 2006; 111: 286-288.

2. Allison DB, Wakely PE, Siddiqui MT, Ali SZ. Nodular fasciitis: A frequent diagnostic pitfall on fine-needle aspiration. Cancer 2016; 125: 20-29.

3. Erickson-Johnson MR, Chou MM, Evers BR et al. Nodular fasciitis: a novel model of transient neoplasia induced by MYH9USP6 gene fusion. Lab Invest 2011; 91: 1427-1433.

4. Goldblum JR, Weiss SW, Folpe AL. Benign Fibroblastic/Myofibroblastic Proliferations, Including Superficial Fibromatoses. In: Enzinger and Weiss's Soft Tissue Tumors. Elsevier Health Sciences, New York 2014; pp. 190-201.

5. Kubota K, Okada S, Maeda T, Matsumoto Y, Sakamoto A, Harimaya K, Saiwai H, Kumamaru H, Oda Y, Iwamoto Y. Extradural nodular fasciitis arising in the spinal canal. Spine 2012; 37: E133-137.

6. Sengupta RP, So SC, Perry RH. Nodular fasciitis: an unusual cause of extradural spinal cord compression. Br J Surg 1975; 62: 573-575.

7. Stanley MW, Skoog L, Tani EM, Horwitz CA. Nodular fasciitis: spontaneous resolution following diagnosis by fine-needle aspiration. Diagn Cytopathol 1993; 9: 322-324. 